\title{
Diffusion Spectrum Imaging
}

National Cancer Institute

\section{Source}

National Cancer Institute. Diffusion Spectrum Imaging. NCI Thesaurus. Code C128124.

A high angular resolution diffusion MRI technique that maps complex tissue structures at the scale of single MRI voxels. 